\title{
COMPORTAMIENTO DE LOS PRONOMBRES EXPLETIVOS EN INGLÉS: ASPECTOS CONTRASTIVOS ENTRE LA PRIMERA Y SEGUNDA LENGUA 1
}

\author{
Yolanda Ruiz de Zarobe \\ Universidad del País Vasco
}

\begin{abstract}
RESUMEN: El objeto del presente artículo es analizar el comportamiento de los pronombres expletivos en la adquisición del inglés como segunda lengua. Analizaremos el proceso adquisitivo desarrollado por estudiantes españoles de inglés, y compararemos los fundamentos que se siguen en la adquisición de una primera y de una segunda lengua. Asimismo, comprobaremos si los pronombres expletivos cumplen un papel fundamental en la adquisición de una segunda lengua como lo hacen en las primeras lenguas.
\end{abstract}

ABSTRACT: The aim of this research is to study the behaviour of expletive pronouns in the acquisition of English as a second language. We will analyze the processes involved in the acquisition of a first and a second language in order to investigate if expletive pronouns play such an important role in the acquisition of English as a second language as they do in first languages.

La teoría lingüística de los principios y parámetros propuesta por Noam Chomsky (1981) afirma que toda lengua se adquiere a través de un conocimiento lingüístico innato o lo que él denomina una Gramática Universal. Chomsky encontró esta explicación al enfrentarse al problema de cómo los niños pueden producir un número ilimitado de oraciones gramaticales cuando su exposición a una lengua es tan limitada y cuando existen a menudo deficiencias en el input recibido. Según él, los niños vienen al mundo equipados con una Gramática Universal que restringe en gran medida las hipótesis que vayan a realizar sobre la lengua. Este conocimiento implícito permite engendrar un número infinito de oraciones y de este conocimiento implícito debe dar cuenta la gramática generativa.

1. Al hablar de la segunda lengua, nos referimos a toda lengua que se adquiere con posterioridad a la lengua materna. 
Uno de los fines de la gramática generativa es enunciar los principios universales que el niño asume innatamente. Uno de estos principios es la teoría de la X', que determina la estructura de la frase. Otro es el Principio de Subyacencia, condición que limita el movimiento de categorías al no permitir que se atraviese más de un nudo cíclico o nudo límite. Estos principios están asociados a una serie de parámetros que adoptan distintos valores y que hacen que exista variación entre las lenguas. Volviendo a nuestro ejemplo anterior, el Principio de Subyacencia tiene un parámetro asociado al mismo; existe variación con respecto a los nudos cíclicos que se adoptan: en francés o en italiano, SN y O' pueden actuar como nudos cíclicos pero no O mientras que en inglés son el SN y la O las categorías que pueden actuar como nudos límite.

La Gramática Universal restringe en gran manera la variación paramétrica que pueda existir para que el niño no tenga que realizar multitud de hipótesis sobre la gramática antes de llegar a la correcta. Cada parámetro posee una serie de propiedades e inicialmente no está marcado. Los estudiantes deben adquirir una lengua a través de datos aportados por su propia lengua, de evidencia positiva y, por lo tanto, el parámetro que no está marcado debe ser el más restrictivo, conformando el núcleo de la gramática. Hay igualmente reglas más específicas, construcciones menos usuales que se aprenden por experiencia y que componen la gramática periférica.

En este estudio, uno de nuestros objetivos ha sido comprobar si la Gramática Universal, que es operativa en la adquisición de una primera lengua sigue funcionando al adquirir una segunda. Estudiaremos si existen procesos similares en la adquisición de una primera y de una segunda lengua, y de ser así, si la Gramática Universal ejerce la misma influencia al adquirir una nueva lengua.

Para nuestro análisis nos hemos centrado fundamentalmente en la adquisición de los pronombres expletivos ingleses, que tradicionalmente forman parte del Parámetro pro-drop o de sujeto nulo, que recientemente ha sido reformulado como Parámetro de argumento nulo (Hyams, 1994, 1996). Básicamente, este parámetro muestra la diferencia que existe en distintas lenguas con respecto a la posibilidad de poseer sujetos fonológicamente nulos.

En lenguas como el español existe la posibilidad de omitir sujetos referenciales:

(1) Vinieron muy tarde.

mientras que esta omisión es imposible en lenguas que no poseen el parámetro pordrop, como es el caso del inglés.

(2) They came very late.

*Came very late.

Además, en inglés existen pronombres expletivos, con valor únicamente gramatical;

(3) It is snowing.

*Is snowing. 
(4) There is a book on the table.

*Is a book on the table.

mientras que éstos no existen en lenguas pro-drop como el español.

(5) Nieva.

*Ello nieva.

(6) Hay un libro en la mesa.

*Ello hay un libro en la mesa.

Según algunos investigadores como Nina Hyams (1986, 1989), el valor de los pronombres expletivos es vital en la adquisición de una primera lengua. Hyams opina que la gramática inglesa de un primer período de la infancia es distinta de la que posteriormente poseen los adultos ingleses ${ }^{2}$. Los niños ingleses a menudo omiten sujetos referenciales. Utilizan frases como:

(7) Want an apple.

(8) Show Mommy that.

En este primer período, que va del año o año y medio a los tres años, vemos que tampoco usan pronombres expletivos y verbos modales. Hyams opina que las lenguas varían con respecto a si CONC se identifica o no con PRO. En las lenguas que poseen el parámetro pro-drop o, como ella lo denomina, el parámetro CONC/PRO, CONC se identifica con PRO al cumplir las mismas propiedades: el poder estar controlado, el poder ser arbitrario y el no poder ser regido. En lenguas como el español CONC se identifica con PRO y la posición de sujeto puede quedar vacía, mientras que en lenguas como el inglés, CONC no se identifica con PRO. Según ella, en el inglés del primer período de la infancia, CONC también se identifica con PRO, y por consiguiente, los niños ingleses omiten, como lo hacen los niños españoles, estos sujetos referenciales. Por otro lado, los pronombres expletivos en lenguas que no poseen el parámetro pro-drop sólo se utilizan por razones gramaticales, ya que la gramática no permite que categorías vacías ocupen la posición de sujeto, pero estos pronombres no poseen ningún contenido semántico. En esta primera etapa el parámetro CONC/PRO junto con el Principio de Elisión del Pronombre, que estipula que se debe anular un elemento pronominal léxico si un elemento pronomi-

2. En este artículo no nos vamos a detener en la exposición de los fundamentos que explican la omisión de sujetos desde un punto de vista gramatical. Aunque algunos estudiosos como P. Bloom (1990), L. Gerken (1991) o Valian (1991) relacionan este proceso con limitaciones en la producción, en esta investigación partimos de la premisa de que la omisión de sujetos tiene una base gramatical. Una exposición detallada de este aspecto aparece en Pierce (1992) y Hyams \& Wexler (1993). 
nal nulo es posible, hacen que los pronombres expletivos se eliminen. Además, Hyams demuestra cómo también se omiten los verbos modales en una etapa inicial. Estos verbos pueden ocupar dos posiciones sintácticas: pueden aparcer en la posición de auxiliar (AUX) como en inglés o pueden situarse dentro del sintagma verbal (SV) como en español. Pero en esta primera etapa ninguna de estas posiciones parece adecuada: los modales no pueden aparecer en AUX, porque entonces regirían PRO y PRO no puede regirse, y tampoco pueden aparecer en SV porque no se pueden analizar como verbos principales, al no poseer morfología verbal. Por lo tanto, los verbos modales también se excluyen en un primer momento. Así pues, los niños ingleses omiten los pronombres referenciales, los pronombres expletivos y los verbos modales durante un cierto período y luego deberán reajustar el parámetro CONC/PRO a través de evidencia positiva para que su gramática se adecúe a la de los adultos.

Nina Hyams opina que los pronombres expletivos provocan el reajuste del parámetro. Estos pronombres, como ya hemos visto, se utilizan en lenguas que no poseen el parámetro CONC/PRO por motivos gramaticales y nunca pragmáticos. No pueden expresar énfasis

(9) She is not coming, he is.

*It's not snowing, it is raining.

o mostrar algún tipo de contraste.

(10) It doesn't seem that we are crazy, it seems that you are.

*It doesn't seem that we are crazy, it seems that you are. ${ }^{3}$

Cuando los niños ingleses perciben estos pronombres, se dan cuenta de su necesidad puramente gramatical y comienzan a utilizarlos. El niño empieza a usar sujetos expletivos más o menos al mismo tiempo que produce sujetos referenciales, lo que demuestra que la gramática se ha reestructurado.

En este estudio, hemos analizado si el orden establecido al adquirir el inglés como primera lengua sigue siendo el mismo cuando se adquiere una segunda. Para ello hemos contado con un corpus de 150 estudiantes españoles de inglés que estaban estudiando en la Universidad Pública de Navarra. Todos ellos formaban parte de un programa de autoaprendizaje, que consistía en la asistencia a clases regladas de inglés durante tres horas a la semana, y la posibilidad de trabajar autónomamente en un centro de autoacceso. Todos los estudiantes, al comienzo del curso escolar, habían realizado una prueba escrita y una entrevista oral con un tutor para conocer su competencia

3. A través de la letra cursiva marcamos los elementos que están acentuados. 
en inglés, y habían sido en consecuencia distribuidos en cinco niveles distintos, similares a los existentes en distintos organismos oficiales. Para nuestra investigación, les pedimos que rellenaran un cuestionario con su historial lingüístico donde contestaban preguntas como los años que habían estado aprendiendo el idioma, los exámenes realizados, las estancias en países de habla inglesa y su conocimiento de otros idiomas, aspectos que eran importantes para tener un conocimiento amplio de todos ellos. Además, nos pareció necesario que realizaran una prueba paralela a la llevada a cabo al inicio del curso, para reafirmar el nivel establecido en cada uno de los casos. Para ello, les pedimos que completaran un test de cloze. Este tipo de pruebas, además de ser fáciles de administrar, muestran unos resultados globales (Oller (1991), Fotos (1991)) $\mathrm{y}$, de este modo, exponen de forma fidedigna el nivel real que se posee en el idioma en cuestión.

Estos 150 alumnos fueron divididos en cinco grupos según su habilidad lingüística,

Nivel 1: 29 alumnos

Nivel 2: 49 alumnos

Nivel 3: 34 alumnos

Nivel 4: 20 alumnos

Nivel 5: 18 alumnos

y se les pidió que realizaran tres pruebas distintas. En primer lugar, debían traducir un total de veintiocho oraciones, que incluían aquellos aspectos del parámetro prodrop o CONC/PRO que nos interesaba analizar. Oraciones como

(11) Había un cuadro muy bonito en tu habitación (pronombre existencial "there").

(12) Es fácil criticar cuando no se conoce a la persona (pronombre expletivo “it").

(13) Juan no puede venir a la reunión (verbo modal “can”) .

Por otro lado, los estudiantes realizaron redacciones escritas de entre 200 y 250 palabras, cuyo tema ya había sido sugerido ("Yesterday I went to the cinema and I saw a very good film”). En tercer lugar, nos pareció importante comprobar su comportamiento en un entorno en el que no tienen tanto tiempo para monitorizar según la terminología krasheniana, por lo que mantuvimos conversaciones orales con todos ellos. La duración de las mismas varió dependiendo de su nivel y de su personalidad, y preferimos realizarlas en pareja cuando los estudiantes se conocían entre sí.

Los resultados en el conjunto de las tres pruebas con respecto al número de errores pro-drop cometidos fueron los siguientes: 
Totales de errores cometidos en cada nivel

$\begin{array}{lrrrrr} & \text { N1 } & \text { N2 } & \text { N3 } & \text { N4 } & \text { N5 } \\ \text { Suj. referenciales } & 29 & 27 & 17 & 4 & 2 \\ \text { Suj. expletivo "it" } & 31 & 49 & 45 & 22 & 15 \\ \text { Suj. existencial "there" } & 3 & 27 & 7 & 1 & 2 \\ \text { Auxiliares } & 57 & 48 & 15 & 9 & 4\end{array}$

Número medio de errores cometidos en cada nivel

$\begin{array}{lrrrrr} & \mathrm{N} 1 & \mathrm{~N} 2 & \mathrm{~N} 3 & \mathrm{~N} 4 & \mathrm{~N} 5 \\ \text { Suj. referenciales } & 1.00 & .55 & .50 & .20 & .11 \\ \text { Suj. expletivo "it" } & 1.07 & 1.00 & 1.32 & 1.10 & .83 \\ \text { Suj. existencial "there" } & .10 & .55 & .21 & .05 & .11 \\ \text { Auxiliares } & 1.97 & .97 & .45 & .45 & .23\end{array}$

\section{Diferencial de errores cometidos en el paso de un nivel a otro}

$\begin{array}{lrrrr} & \mathrm{D} 12 & \mathrm{D} 23 & \mathrm{D} 34 & \mathrm{D} 45 \\ \text { Suj. referenciales } & -.45 & -.05 & -.30 & -.09 \\ \text { Suj. expletivo "it" } & -.07 & .32 & -.22 & -.27 \\ \text { Suj. existencial "there" } & .45 & -.35 & -.16 & .06 \\ \text { Auxiliares } & -1.00 & -.52 & 0.00 & -.22\end{array}$

Los resultados de las tres pruebas demuestran cómo en el caso de los sujetos referenciales y de los auxiliares, el número de errores pro-drop disminuye a medida que el nivel aumenta, lo que puede parecer lógico. Sin embargo, en el caso de los pronombres expletivos, el número de errores, lejos de disminuir, aumenta en los niveles intermedios para volver a decrecer en los superiores. Aun así, el número de errores pro-drop en los niveles superiores es significativamente mayor en el caso de los pronombres expletivos que en el resto de variables, lo que nos va a hacer cuestionarnos su importancia en la adquisición de segundas lenguas. Aunque éstos juegan un papel pri- 
mordial en la adquisición de una primera lengua, provocando el reajuste del parámetro pro-drop, vemos cómo no pueden causar el reajuste del mismo en la adquisición del inglés como segunda lengua, ya que los estudiantes consiguen controlarlos con posterioridad. A nuestro modo de ver, la lentitud con la que los pronombres expletivos se adquieren puede explicarse bajo una perspectiva contrastiva. Los estudiantes españoles no poseen sujetos expletivos en su lengua base por el Principio de Elisión del Pronombre y por ello, su comportamiento con respecto a ellos es bastante limitado, cometiendo numerosos errores, y su adquisición tardía.

Por otro lado, el proceder de ambos pronombres expletivos es desigual. Bajo un enfoque transferencial, los dos tipos de pronombres deberían comportarse de igual manera, por no aparecer ninguno de ellos en la lengua base. Sin embargo, el número de errores en el caso del existencial "there" es significativamente menor. Según nuestra opinión, esta diferencia se puede deber a la propia naturaleza semántica del existencial, que resulta menos complejo para los estudiantes, que ya conocen el locativo "there", al ser uno de los primeros en adquirirse. Por otro lado, el existencial "there" parece formar una unidad con el verbo copulativo "be", ya que los estudiantes aprenden ambas partículas conjuntamente, vinculándolas, y de ahí que se hayan cometido menos omisiones.

Así pues, comprobamos cómo los pronombres expletivos no siguen el orden decreciente del resto de las características. Esto nos va a hacer cuestionarnos la hipótesis que opina que los procesos adquisitivos en una primera lengua son similares a los existentes en la adquisición de una segunda lengua. Según la hipótesis de Hyams para primeras lenguas, los pronombres expletivos se adquieren con cierta anterioridad a los sujetos referenciales y a los auxiliares, provocando el reajuste del parámetro pro-drop. Si transpasamos esta hipótesis a segundas lenguas (Hilles, 1986), los estudiantes españoles de inglés deberían comenzar a utilizar obligatoriamente los sujetos referenciales y a controlar adecuadamente la forma y contenido de los verbos auxiliares una vez se percatan de la existencia de los pronombres expletivos, que evidencian que en inglés es necesario poseer una categoría con función de sujeto, aunque ésta tenga un valor puramente gramatical. En nuestra investigación hemos comprobado cómo el número de errores pro-drop con respecto a los sujetos expletivos aumenta a lo largo de los niveles, por lo que no podemos confirmar que su adquisición preceda a la de los sujetos referenciales o a la de los auxiliares. Es más, parece que su adquisición se ve postergada hasta que ambas categorías han sido controladas.

\section{Conclusiones}

Nuestro estudio ha demostrado cómo el papel de los pronombres expletivos ingleses no es tan importante en la adquisición de segundas lenguas como en un principio podría haberse previsto. Estos pronombres no provocan el reajuste del parámetro 
pro-drop, sino que su adquisición se retrasa hasta que otras categorías tales como los sujetos referenciales y los auxiliares han sido adquiridas. Este hecho va a hacer que debamos revisar el orden de adquisición de los distintos elementos que tradicionalmente forman parte del parámetro pro-drop.

\section{Bibliografía}

BLOOM, P., (1990): “Subjectless sentences in child language”, Linguistic Inquiry, 21, pp 491-504.

CHOMSKY, N., (1981): Lectures on government and binding, Dordrecht: Foris.

CHOMSKY, N., (1982): Some concepts and consequences of government and binding, Cambridge Mass: MIT Press.

CHOMSKY, N., (1986): Barriers. Cambridge, Mass: MIT Press.

FOTOS, S., (1991): "The cloze test as an integrative measure of EFL proficiency: A substitute for essays on college entrance examinations?". Language Learning, 41, pp. 313-336.

GERKEN, L., (1991): “The Metrical basis for children's subjectless sentences”, Journal of Memory and Language, 30, pp. 431-451.

HYAMS, N. M., (1986): Language acquisition and the theory of parameters. Dordrecht: D. Reidel Publishing Company.

HYAMS, N. M., (1989): “The null subject parameter in language acquisition”, en O. Jaeggli y J. K. Safir, The Null Subject parameter, Dordrecht: Kluwer, pp. 215-238.

HYAMS, N. M., (1994): "V2 null arguments and COMP projections". En Hoekstra. T y B. Schwartz (eds), Language acquisition studies in generative grammar, Amsterdam: John Benjamins.

HYAMS, N. M. y WEXLER, K., (1993): “On the Grammatical Basis of Null Subjects in Child Language”, Linguistic Inquiry, 24, pp. 421-459.

INGHAM, R., (1992): “The optional subject phenomenon in young children's English: A case study", Journal of Child Language, 19, pp. 133-151.

KRASHEN, S. D., (1984): Second language acquisition and second language learning, New York: Oxford University Press.

LASNIK, H., (1992): "Case and expletives: Notes toward a parametric account", Linguistic Inquiry, 23, pp. 381-405.

OLLER, J., Jr., (1991): “Foreign language testing: I: Its breadth; II: Its depth”. ADFL Bulletin 22; 23, pp. 33-38/5-13.

PIERCE, A., (1992): Language acquisition and syntactic theory: A comparative study of French and English child grammars, Dordrecht: Kluwer.

RUIZ DE ZAROBE, Y., (1996): La actuación del parámetro pro-drop en la adquisición del inglés como segunda lengua, tesis doctoral. Universidad del País Vasco. 
RUIZ DE ZAROBE, Y., (1996): "Expletive pronouns and the readjustment of the prodrop parameter". The Forum of Phi Sigma Iota, International Foreign Language Honor Society, University of Nevada, 18 (1), pp. 9-10.

RUIZ DE ZAROBE, Y., (1998): "El parámetro pro-drop y la adquisición de segundas lenguas". ITL: Review of Applied Linguistics, pp. 121-122.

RUIZ DE ZAROBE, Y., (1998): "Uniformidad morfológica y adquisición de sujetos en inglés lengua extranjera". Langues et Linguistique, 24.

VALIAN, V., (1991): "Syntactic subjects in the early speech of American and Italian children", Cognition, 40, pp. 21-81. 